\title{
Distributed Subchannel Assignment in a Multiuser MIMO Relay
}

\author{
Tiina Heikkinen \\ Department of Computer Science \\ P.O. Box 68 \\ FIN-00014 University of Helsinki, Finland \\ tiina.heikkinen@cs.helsinki.fi
}

\author{
Ari Hottinen \\ Radio Communications CTC \\ Nokia Research Center \\ P.O. Box 407, FIN-00045 Nokia Group, Finland \\ ari.hottinen@nokia.com
}

\begin{abstract}
The paper studies a relay network that comprises multiple source nodes, one common multi-antenna relay node and multiple destination nodes. The transmitting nodes (source and relay) have access to channel information and to some interference-related information. A noncooperative game model is used to address the distributed resource allocation problem among different source nodes. Qualityof-Service-aware source nodes opportunistically select their transmission resources while a multi-antenna channel-aware relay node assigns the resources at the relay in a way that maximizes the sum of utilities at the destination nodes. The beam allocation game is a potential game for which the implicit joint objective (potential) of the source nodes formalizes a trade-off between the sum of individual utilities and the sum of congestion costs. Numerical examples illustrate the performance of opportunistic channel allocation.
\end{abstract}

\section{Keywords}

Relay network, resource allocation, noncooperative game theory, MIMO, Beamforming

\section{INTRODUCTION}

Relays have been used recently in diverse applications in wireless systems, including large-scale broadcast networks such DVB and their use has been proposed recently e.g. in IEEE 802.16 standardization for OFDMA systems. Relay nodes improve communication between a source and a destination by decomposing one link into one or multiple shorter links.

In this paper, we address a (partially) distributed resource allocation problem in a multi-channel relay involving multiple source nodes, one multi-antenna relay node and one destination node. The source nodes compete for the use of the spatial channels (beams) between the source and the relay, avoiding collisions via feedback control. The multiantenna relay node is seen as a network element that has access to channel state information. Its task is to reassign

Permission to make digital or hard copies of all or part of this work for personal or classroom use is granted without fee provided that copies are not made or distributed for profit or commercial advantage and that copies bear this notice and the full citation on the first page. To copy otherwise, to republish, to post on servers or to redistribute to lists, requires prior specific permission and/or a fee.

GameComm '07, October 22, 2007, Nantes, France

Copyright 2007 ICST 978-963-9799-00-4. the resources at the relay so that network performance is optimized. The relay may reassign a received subchannel (beam) to a different output subchannel (beam), thereby controlling the performance of selected sources.

In single source, single destination case, with one antenna at relay, related work has established algorithms for determining the subcarrier reassignment in connection with OFDM or OFDMA systems $[4,2,5]$. In this paper, like in [5], we extend these to the case of multiple source nodes competing for a limited number of resources at the relay node. In this paper, the competition is applied spatial channels both at relay node input and relay node output whereas [5] considers a single-antenna system. The approach to distributed resource allocation is based on noncooperative game theory [6]. Assuming noncooperative (distributed) resource allocation, the source nodes only need local channel quality information to decide the individually optimal allocation. In a game model of distributed resource allocation, QoS-aware nodes are assumed to assign the subchannels (beams) in a way that maximizes individual utilities (e.g. in terms of signal-to-noise ratio or capacity).

The game formulation is similar in this paper and in [5]. However, in this paper the game problem is elaborated as a potential game [3] for which the implicit joint objective (potential) of the noncooperative source nodes can be stated in closed form. The potential function of the beam allocation game suggests that the game problem corresponds to a problem to optimize a trade-off between the sum of individual utilities and the sum of congestion costs. Furthermore, the game problem is formalized as sequential game of imperfect information [7].

The paper is structured as follows. Section 2 formalizes the multi-antenna signal model for a multiuser two-hop relaying scheme. Section 3 studies a potential game model for distributed beam allocation. For simplicity, transmit power is assumed to be constant. Numerical examples are discussed in section 4 . Section 5 concludes.

\section{SIGNAL MODEL}

In our system model, each source and destination node have only one transmit and receive antenna, but the relay node in between has $N_{t}$ antenna elements in both reception and transmission. In the two-hop system transmission and reception occur at different times (or at different frequency) so as to avoid self-interference at relay. We assume that there is the same number of beams in both links, i.e. $p, p^{\prime} \in$ $\{1, \ldots, P\}$ and that these do not depend on the channels. 
However, the nodes are channel-aware in the sense that they can measure the effective channel quality that results from using any prescribed beam indices. The interpretation is that there are $P$ random beams in each link, and these are used sequentially so that at any given time only one of them is active.

The signal received from source $m$ at relay using receive beam $p^{\prime}$ is given by

$$
z_{m}\left[p^{\prime}\right]=\mathbf{w}_{p^{\prime}}^{H} \mathbf{h}_{m} x[m]+n_{2}\left[p^{\prime}\right],
$$

where noise at relay $n_{2}$ is assumed to be iid circular Gaussian (with variance $\sigma_{2}^{2}$ ), $x[m]$ is the transmitted symbol, and $\mathbf{w}_{p}$ represents the receive beam at the relay. Correspondingly, since we consider an amplify-and-forward relay, the relay transmits the symbol $z_{m}\left[p^{\prime}\right]$ and the destination node receives

$$
y_{m}[p]=\sqrt{\lambda\left[p^{\prime}\right]}\left(\mathbf{v}_{p}^{H} \mathbf{e}_{m} z[m]+n_{3}[p]\right),
$$

where $n_{3}$ is noise at destination (with variance $\sigma_{3}^{2}$ ) and $N_{t^{-}}$ dimensional vectors $v_{p}$ and $\mathbf{e}_{m}$ represent the $p$ th transmit beam at relay and the channel between relay and $m$ th destination, respectively. Possible power allocation at relay is modelled with $\lambda\left[p^{\prime}\right]$. For example, with

$$
\lambda\left[p^{\prime}\right]=\left(\left|d_{m}\left[p^{\prime}\right]\right|^{2}+\sigma_{2}\left[p^{\prime}\right]^{2}\right)^{-1 / 2},
$$

the transmit power at relay is normalized to unity.

To simplify notations in what follows, we write

$$
d_{m}[p]=\left|\mathbf{w}_{p}^{H} \mathbf{h}_{m}\right|^{2}
$$

and

$$
q_{m}[p]=\left|\mathbf{v}_{p}^{H} \mathbf{e}_{m}\right|^{2} .
$$

A signal that is transmitted from the source is received at relay using beam with index $p^{\prime}$. Thereafter, it is retransmitted using amplify-and-forward protocol using a transmit beam with index $p$. Thus, the destination node (Node 3 ) receives

$$
y_{m}[p]=q_{m}[p] \lambda\left[p^{\prime}\right]\left(d_{m}\left[p^{\prime}\right] x+n_{2}\left[p^{\prime}\right]\right)+n_{3}[p] .
$$

Hence, indices $p$ and $p^{\prime}$ allow us to model beam reassignment at relay. This is, in what follows, captured by a permutation matrix $\mathbf{T}$. The element $t_{p, p^{\prime}}=1$ if receive beam $p^{\prime}$ is assigned to transmit beam $p$ at relay output, otherwise $t_{p, p^{\prime}}=0$.

For a given assignment $\left(p, p^{\prime}\right)$ the received signal power may be easily computed to be for source-destination pair $m$

$$
\mathrm{S}^{m}\left[p, p^{\prime}\right]=\left|d_{m}\left[p^{\prime}\right] \lambda[p] q_{m}[p]\right|^{2} .
$$

Likewise, the noise power is

$$
\mathrm{N}^{m}\left[p, p^{\prime}\right]=\sigma_{3}[p]^{2}+\left|\lambda[p] q_{m}[p]\right|^{2} \sigma_{2}\left[p^{\prime}\right]^{2}
$$

assuming that an amplify-and-forward relay is used. Consequently, the effective signal-to-noise ratio (SNR) at destination for the assignment $\left(p, p^{\prime}\right)$ is

$$
\gamma_{3}^{m}\left[p, p^{\prime}\right]=\mathrm{S}_{m}\left[p, p^{\prime}\right] / \mathrm{N}_{m}\left[p, p^{\prime}\right]
$$

The link-specific signal-to-noise-ratio in the $p^{\prime}$ th received beam for the source-relay link, and for the $p$ th transmit of the relay-destination link, are defined as

$$
\gamma_{r, m}\left[p^{\prime}\right]=\left|d_{m}\left[p^{\prime}\right]\right|^{2} / \sigma_{2}\left[p^{\prime}\right]^{2},
$$

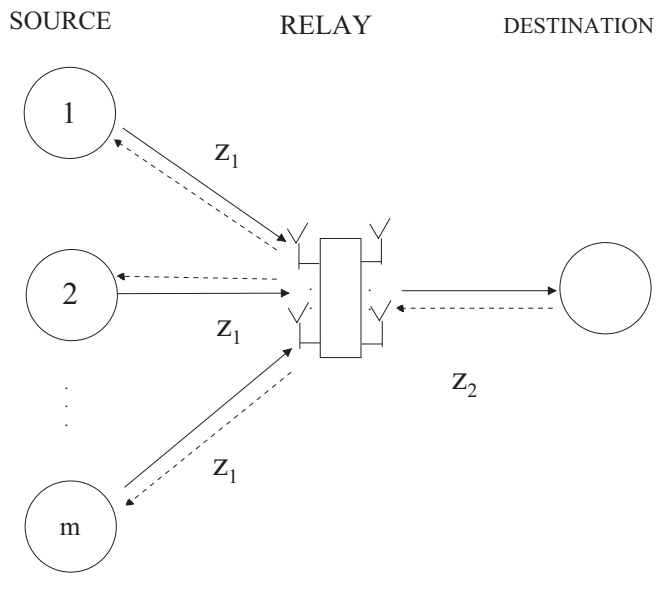

Figure 1: Network with $m$ source nodes, one multiantenna relay node and one destination node. Feedback messages between relay and each source, and destination and relay, are denoted by $z_{1}$ and $z_{2}$, respectively.

and

$$
\gamma_{d, m}[p]=\left|q_{m}[p]\right|^{2} / \sigma_{3}[p]^{2} .
$$

Using these notations, we can rewrite SNR at the destination node as

$$
\gamma_{3}^{m}\left[p, p^{\prime}\right]=\frac{\gamma_{r, m}\left[p^{\prime}\right] \gamma_{d, m}[p]}{\gamma_{r, m}\left[p^{\prime}\right]+\gamma_{d, m}[p]+1} .
$$

In a multiuser system there are $m$ source nodes, each accessing a respective destination via one of the $P$ input and output beams of the same relay. In the considered case, the same beam can be assigned only to one user at a time. To account for this, and aid distributed assignment, the relay node signals the source nodes vector $\mathbf{z}_{1} \in\{0,1\}^{P}$, which contains crude information pertaining to interference power in different beams at the receiver of the relay node. In the simplest case, the relay sets $\mathbf{z}_{1}\left[p^{\prime}\right]=1$ if beam $p^{\prime}$ is feasible for the considered source node, otherwise $\mathbf{z}_{1}\left[p^{\prime}\right]=0$. The feasibility can be determined by comparing the $\gamma_{3}\left[p^{\prime}\right]$ at destination to some threshold or simply by setting $\mathbf{z}_{1}\left[p^{\prime}\right]=0$ if beam $p^{\prime}$ is already assigned to another user. Analogously, the destination node signals the relay node $\mathbf{z}_{2} \in\{0,1\}^{P}$, as shown in Fig. 1.

The interference information $\mathbf{z}_{1}$ and $\mathbf{z}_{2}$ is used at the relay node to modify the effective noise power in model (3) with

$$
\sigma_{2}^{2}\left[p^{\prime}\right] \leftarrow\left(1-\mathbf{z}_{1}\left[p^{\prime}\right]\right) \sigma_{0}^{2}+\mathbf{z}_{1}\left[p^{\prime}\right] \sigma_{2}^{2}\left[p^{\prime}\right]
$$

and

$$
\sigma_{3}^{2}[p] \leftarrow\left(1-\mathbf{z}_{2}[p]\right) \sigma_{0}^{2}+\mathbf{z}_{2}[p] \sigma_{3}^{2}[p],
$$

where we set $\sigma_{0}=\infty$ if a beam is punctured or unavailable for the user of interest. 


\section{DISTRIBUTED BEAM ALLOCATION}

A sequential game is one in which players make decisions following a predefined order, and in which at least some players can observe the moves of players who preceded them. The beam allocation game can be modelled as a sequential game as follows. First, at the source-destination link there are $m$ source nodes competing for a limited number of subchannels (beams). The relay node can observe the choices of the source nodes. At the relay-destination link, the relay is assumed to choose an optimal beam allocation over users given channel information. It is not realistic to assume that the source nodes are able to observe the channel quality at the relay-destination link and the choices of the relay. Thus, the beam allocation game is one of imperfect information that can be modelled as a Bayesian game. In a Bayesian game a player (source) knows neither the other players' (relay's or the other source nodes') private (local) information not his strategy choices [6, 7]. The sequential beam allocation game can be outlined as a dynamic game with three stages:

1. Nature chooses the "type" of the relay node, specifying the subchannels available at the relay-destination link;

2. Noncooperative source nodes decide on the beam allocation at the source-relay link;

3. Relay node decides on the beam allocation at the relaydestination link, observing its "type" and the beam allocation in the preceding stage.

Formally, let $\tau_{1}^{i}[p]=1$ if $i$ gets beam $p$ and let $\tau_{1}^{i}[p]=0$ otherwise. For the source relay-link, the source nodes decide on the beam allocation as noncooperative players. Let $\tau_{2}^{i}\left[p^{\prime}\right]=1$ if $i$ gets subchannel $p^{\prime}$ and let $\tau_{2}^{i}\left[p^{\prime}\right]=0$ otherwise. Assuming $i$ th source obtains assignment $\left(p, p^{\prime}\right)$, the utility of source nodes $i=1, \ldots, m$, first assuming for simplicity perfect information, can be defined as

$$
\gamma_{3}^{i}\left[p, p^{\prime}\right] \tau_{1}^{i}[p] \tau_{2}^{i}\left[p^{\prime}\right]=\frac{\gamma_{12}^{i}\left[p^{\prime}\right] \gamma_{23}[p]}{\gamma_{12}^{i}\left[p^{\prime}\right]+\gamma_{23}[p]+1} \tau_{1}^{i}[p] \tau_{2}^{i}\left[p^{\prime}\right] .
$$

As $\gamma_{12}^{i}\left[p^{\prime}\right]$ depends of the source index $i$ and each source-torelay channel is independent of each other, the source nodes have different valuations for a given allocation $\left(p, p^{\prime}\right)$.

In a distributed noncooperative system each source maximizes the expected value of its individual utility function (7) taking as given the strategy choices of other source nodes. In general each node may choose more than one subchannel for each link. Let $\mathcal{I}$ denote the set of subchannels available to all source nodes and let $\mathcal{J}$ denote the set of subchannels at relay. As an example, the utility of source $i$ could be defined as the sum

$$
u^{i}\left(\tau_{1}^{i}, \tau_{2}^{i}\right)=\sum_{p \in \mathcal{I}} \sum_{p^{\prime} \in \mathcal{J}} \gamma_{3}^{i}\left[p, p^{\prime}\right] \tau_{1}^{i}[p] \tau_{2}^{i}\left[p^{\prime}\right]
$$

where $\tau_{1}^{i}=\left\{\tau_{1}^{i}[p]\right\}$ and $\tau_{2}^{i}=\left\{\tau_{2}^{i}\left[p^{\prime}\right]\right\}$.

Fairness in a distributed channel-allocation game could be achieved via absolute resource constraints, e.g. requiring for each $i=1, . ., m$ :

$$
M \leq \sum_{p^{\prime} \in \mathcal{J}} \tau_{2}^{i}\left[p^{\prime}\right] \leq H,
$$

where $M$ denotes the minimum number of subchannel pairs to be assigned to a given source and $H$ denotes a maximum number of subchannel pairs to be assigned. At the relay, fairness can be formalized by analogous resource constraints:

$$
M \leq \sum_{p \in \mathcal{I}} \tau_{1}^{i}[p] \leq H
$$

DeFINITION 3.1. A distributed subchannel allocation game can be defined by $m+1$ problems where each source $i=$ $1, \ldots, m$ maximizes the expected value of utility $u^{i}$ given in equation (8) with respect to $\tau_{2}^{i}$ subject to ith constraint (9) taking as given the allocation at the relay $\tau_{1}=\left\{\tau_{1}^{i}\right\}$; the relay node is the $m+1$ st player solving

$$
\max _{\left\{\tau_{1}^{i}\right\}} \sum_{i} u^{i}\left(\tau_{1}^{i}, \tau_{2}^{i}\right)
$$

To define a solution concept to the imperfect information game in Definition 3.1, the concept of a Bayesian Nash equilibrium [6] can be applied. However, due to the simple form of the utility functions in (4), the imperfect information game can be reduced to a game of perfect information as follows. Consider source node $i$ deciding on its subchannel allocation in the absence of knowledge of the subchannel allocation at the relay. Assume for example, that the source nodes choose their strategies asynchronously in a random order. The "type" information regarding the relay node then can be thought of as including both the update order and the channel information for each update order. Without loss of generality, assume source node $i$ is the first to choose its beam allocation, and the channel at relay can be either "good" or "average", implying $E\left[\gamma_{d, m}[p]\right]>0$. Independently of the channel quality at the relay, it can be seen from (4) that the optimal strategy of node $i$ is to pick its $M$ best beams. Assuming $E\left[\gamma_{d, m}[p]\right]>0$, the preference ordering of subchannels $\left\{p^{\prime}\right\} \in \mathcal{J}$ implied by $\gamma_{3}^{i}\left[p, p^{\prime}\right]$ is the same as that implied by $\gamma_{r, m}^{i}\left[p^{\prime}\right]$. To simplify the distributed allocation problem, a simplified utility of source $i$ can be defined as:

$$
u_{s}^{i}\left(\tau_{2}^{i}\right)=\sum_{p^{\prime}} \gamma_{r, m}^{i}\left[p^{\prime}\right] \tau_{2}^{i}\left[p^{\prime}\right]
$$

where $\tau_{2}^{i}=\left\{\tau_{2}^{i}\left[p^{\prime}\right]\right\}$.

DEFINITION 3.2. A simplified subchannel allocation game with perfect information can be defined by $m+1$ problems where each source $i=1, \ldots, m$ maximizes simplified utility $u_{s}^{i}\left(\tau_{2}^{i}\right)$, given in equation (12), subject to ith constraint (9) (Stage 1) and the relay node solves problem (11) with utility $u_{i}$ in (8) (Stage 2).

Letting $\tau_{2}^{-i}$ and $\tau_{1}^{-i}$ denote the subchannel allocations of users other than $i$ at the source and relay, respectively, Nash equilibrium $\tau_{2}^{i}, i=1, \ldots, m$ in Stage 2 of the simplified sequential game can be defined by inequalities:

$$
u_{s}^{i}\left(\tau_{2}^{i}, \tau_{2}^{-i}\right) \geq u_{s}^{i}\left(\tau_{2}^{i \prime}, \tau_{2}^{-i}\right), \quad i=1, \ldots, m
$$

where $\tau_{2}^{i \prime} \neq \tau_{2}^{-i}, i=1, \ldots, m$.

The solution to the relay's problem (11) is discussed in [5], where it is shown that the related assignment problem can be solved by pairing subchannels of the same rank (ordered by QoS) with each other.

\section{Potential Game}

Recently, [3] has observed that many resource allocation problems in congested networks can be modelled as a potential game. The beam allocation game is another example 
of a potential game, with an implicit joint objective function (potential):

Proposition 3.3. Consider the game defined by utility functions $u_{s}^{i}, i=1, \ldots, m$ in (12), assuming for simplicity that $\tau_{2}^{i}\left[p^{\prime}\right], i=1, \ldots, m$, for each subchannel $p^{\prime}$ is continuous. The potential function $\Phi$ corresponding to this game can be stated as:

$$
\Phi=\sum_{i} u_{s}^{i}-L \sum_{p^{\prime}} \sum_{i<j} \tau_{2}^{i}\left[p^{\prime}\right] \tau_{2}^{j}\left[p^{\prime}\right]+\sum_{i} \lambda_{2}^{i}\left(H-\sum_{p^{\prime}} \tau_{2}^{i}\left[p^{\prime}\right]\right)
$$

where $L$ is a large number and $\lambda_{2}^{i}$ is the Lagrangean multiplier for ith constraint (9).

Proof. The first order optimality condition for maximizing $\Phi$ with respect to $\tau_{2}^{i}\left[p^{\prime}\right]$ can be stated as

$$
\gamma_{12}^{i}\left[p^{\prime}\right]-L \sum_{j \neq i} \tau_{2}^{j}\left[p^{\prime}\right]-\lambda_{2}^{i} \geq 0
$$

Recall the definition of the interference power in (5). Whenever $\tau_{2}^{j}\left[p^{\prime}\right]=1$ for $j \neq i$, the congestion signal $\mathbf{z}_{1}\left[p^{\prime}\right]=0$ to source $i$, and the value of the interference power for source $i$, $\sigma_{2}^{2}\left[p^{\prime}\right]$, becomes $\infty$. Thus, inequality (15) coincides with the condition for $\tau_{2}^{i}\left[p^{\prime}\right]$ being the best-reply of $i$ to the strategy choices of nodes $j \neq i$. Since the best-reply of each source node $i$ can be obtained by maximizing $\Phi$ subject to (9), $\Phi$ can ce considered as the implicit joint objective in the beam allocation game.

The potential function $\Phi$ in (14) models a tradeoff between the sum of utilities and the sum of congestion costs. The optimal solution to maximizing simple linear utility $u_{s}^{i}$ subject to $i$ th constraint (9) is to pick $H$ best available beams. Given congestion information, each source node makes an efficient decision. Similarly, it can be shown that the potential function $\tilde{\phi}$ for the game problem in Definition 3.2 can be written as:

$$
\begin{aligned}
\tilde{\phi}= & \sum_{i} u_{i}-L \sum_{p^{\prime}} \sum_{i<j} \tau_{2}^{i}\left[p^{\prime}\right] \tau_{2}^{j}\left[p^{\prime}\right] \\
& +\sum_{i} \lambda_{2}^{i}\left(H-\sum_{p^{\prime}} \tau_{2}^{i}\left[p^{\prime}\right]\right)+\sum_{i} \lambda_{1}^{i}\left(H-\sum_{p} \tau_{1}^{i}[p]\right) \\
& +\sum_{i} \tilde{\lambda}_{1}^{i}\left(\sum_{p} \tau_{1}^{i}[p]-M\right)
\end{aligned}
$$

where $\lambda_{1}^{i}$ and $\tilde{\lambda}_{1}^{i}$ denote the Lagrangean multipliers for $i$ th resource constraints in (10).

The game defined by payoff functions (8) or (12) (with constraints (9)) is a potential game. Recent work in $[3,1]$ on potential games has specified conditions under which a Nash equilibrium can be found using a simple greedy iteration where each user chooses its individually optimal allocation given the strategy choices of other nodes. For example with utilities $u_{s}^{i}, i=1, \ldots, m$ a convergent distributed resource allocation algorithm can be defined as follows:

Definition 3.4. Assume the source nodes update their subchannel strategies asynchronously in a random order. A distributed subchannel allocation algorithm can be defined for th iteration as the problem of source $i$ :

$$
\max _{\tau_{2}^{i}(t)} u_{s}^{i}\left(\tau_{2}^{i}(t)\right)
$$

subject to ith constraint (9).

\section{PERFORMANCE}

The proposed models for spatial channel allocation at relay are are evaluated below numerically. We assume that the elements of $1 \times N_{t}$ dimensional source-to-relay channels of each user, and the $N_{t} \times 1$ dimensional relay-to-destination channel are each iid Rayleigh distributed. The two sets $\left\{\mathbf{w}_{p}\right\}$ and $\{\mathbf{e}\}$, comprise each an unitary set of $N_{t}$-dimensional beams. The average $S N R$ of each beam is set to $6 \mathrm{~dB}$ in both relay links and performance is evaluated for different cases, with $N_{t}=1,2,4,8,16$ antennas or beams in both relay input and output.

The users in the source-to-relay link select the beam indices sequentially from the set of available beams (cf. Definition 3.4 and equation (12)). This set is known to each source via vector $\mathbf{z}_{1}$, which is broadcasted from the relay node to following source node after a user has reserved her beams or antennas at the relay node. In the reservation process the first user is in a preferred position, since she selects the indices of $P_{1}$ best beam from all $P$ available beams. The next source selects the best beam from $P-P_{1}$ remaining beams, and so on. The last user in the selection process is left with $P_{m}$ beams, i.e. those that no other source appreciated. Hence, first source obtains largest selection diversity gain, and the last has no selection diversity gain. As explained above, with four users $P_{m}=4, \forall m$ and with sixteen users $P_{m}=1, \forall m$, and in both cases $\sum_{m} P_{m}=P$. This applies to the source-to-relay link only. With sixteen users, each user is allocated one distinct beam in both source-torelay and relay-to-destination link. In the experiment with four users, each user has four beams that are solely used by them.

Having selected the relay receive antennas or beams, as described above, the relay determines the associated output antenna or beam indices. When this is done using the assignment method (legend 'with assignment' in Fig. 2 and 3) the relay transmits via the optimal channel-dependent relay input and relay output antennas pairs by solving equation (11) that leads to network optimal capacity. For comparison, the output antenna may also be predefined e.g. so that an input antenna/beam $p$ is always connected to relay output antenna/beam $p$. Performance using this approach is labelled as with legend 'no assignment' in Figs. 2 and 3.

The effect of optimal assignment at the multi-antenna relay is depicted most clearly with the solid lines in Fig. 2. Therein (labeled with "No Assignment"), the relay transmits to an arbitrary destination. Optimal assignment refers to the optimal assignment of output beams at relay. This is obtained as the solution to problem (11), taking as given distributed source-to-relay subchannel allocation. With only one relay antenna, assignment is obviously irrelevant. With increasing $N_{t}$ both approaches benefit from multi-antenna gain at relay input, but only if assignment is used is the relay-to-destination link useful.

Figure 3 shows the capacities for different users, reflecting fairness. Clearly, not using optimal assignment results in a more equal resource allocation at the cost of average capacity.

\section{CONCLUSION}

The multiuser beam or antenna assignment problem in a multi-antenna amplify and forward relay is formulated as a distributed resource allocation problem. Each user is a 


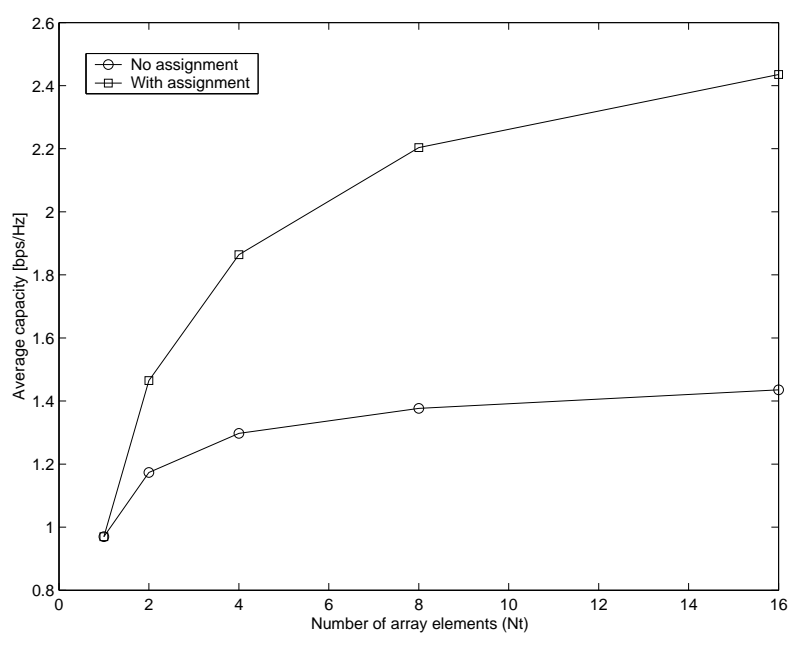

Figure 2: Average capacity at destination nodes with varying $N_{t}$.

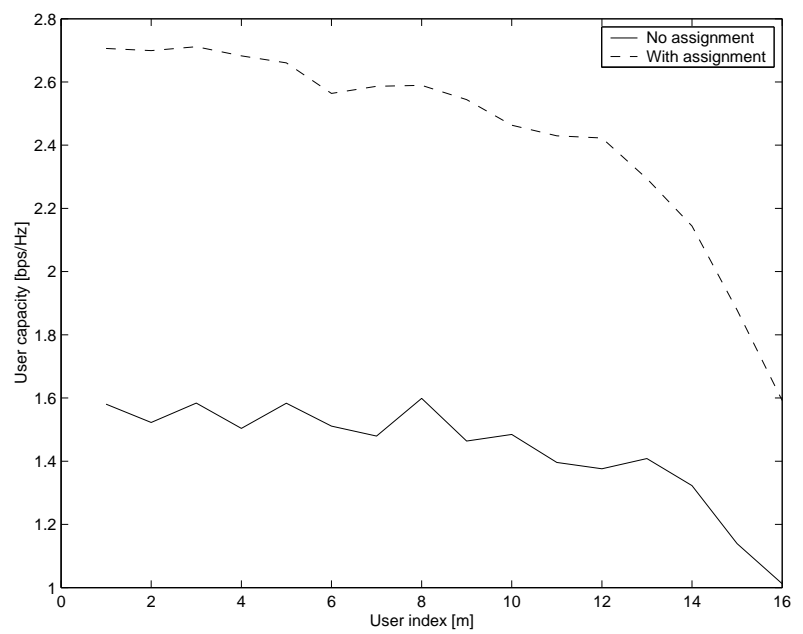

Figure 3: Comparison of the capacity at destination for different users when $N_{t}=16$. noncooperative player in a resource allocation game formulated as a potential game. The potential function for the beam allocation game captures a trade-off between the sum of utilities and congestion costs. Numerical examples, assuming constant transmit power, illustrate the performance of distributed beam assignment; opportunistic channel allocation implies a trade-off between the average capacity and fairness.

\section{ACKNOWLEDGEMENTS}

The work is supported in part by the STREP project No. IST-026905 (MASCOT) within the sixth framework programme of the European Commission

\section{REFERENCES}

[1] E. Altman and Z. Altman. S-modular games and power control in wireless networks. IEEE Tr. Automatic Control, 48(5):839-842, May 2003.

[2] I. Hammerström and A. Wittneben. Joint power allocation for nonregenerative MIMO-OFDM relay links. In Proc. International Conference on Acoustics, Speech, and Signal Processing (ICASSP), Toulouse, France.

[3] T. Heikkinen. A potential game approach to distributed power control and scheduling. Comput. Networks, 50(13):2295-2311, 2006.

[4] A. Hottinen and T. Heikkinen. Subchannel assignment in OFDM relay nodes. In Proc. CISS 2006, Princeton, NJ, USA.

[5] A. Hottinen and T. Heikkinen. Distributed subchannel assignment in an OFDMA relay. In Proc. ValueTools 2007, Nantes, France, Oct 23-24 2007.

[6] A. Mas-Colell, M. Whinston, and J. Green. Microeconomic Theory. Oxford University Press, 1995.

[7] M. Osborne and A. Rubinstein. A course in game theory. MIT Press, 1994. 